\title{
Development of a Modified Vacuum Cleaner for Lunar Surface Systems
}

\author{
Katherine P. Toon ${ }^{1}$ \\ NASA Lyndon B. Johnson Space Center, Houston, TX 77058 \\ Steve A. Lee ${ }^{2}$ \\ MEI Technologies, Inc., Houston, TX, 77058 \\ and \\ Rachel D. Edgerly ${ }^{3}$ \\ NASA Lyndon B. Johnson Space Center, Houston, TX 77058
}

The National Aeronautics and Space Administration (NASA) mission to expand space exploration will return humans to the Moon with the goal of maintaining a long-term presence. One challenge that NASA will face returning to the Moon is managing the lunar regolith found on the Moon's surface, which will collect on extravehicular activity (EVA) suits and other equipment. Based on the Apollo experience, the issues astronauts encountered with lunar regolith included eye/lung irritation, and various hardware failures (seals, screw threads, electrical connectors and fabric contamination), which were all related to inadequate lunar regolith mitigation.

A vacuum cleaner capable of detaching, transferring, and efficiently capturing lunar regolith has been proposed as a method to mitigate the lunar regolith problem in the habitable environment on lunar surface. In order to develop this vacuum, a modified "off-the-shelf" vacuum cleaner will be used to determine detachment efficiency, vacuum requirements, and optimal cleaning techniques to ensure efficient dust removal in habitable lunar surfaces, EVA spacesuits, and air exchange volume.

During the initial development of the Lunar Surface System vacuum cleaner, systematic testing was performed with varying flow rates on multiple surfaces (fabrics and metallics), atmospheric (14.7 psia) and reduced pressures (10.2 and 8.3 psia), different vacuum tool attachments, and several vacuum cleaning techniques in order to determine the performance requirements for the vacuum cleaner. The data recorded during testing was evaluated by calculating particulate removal, relative to the retained simulant on the tested surface. In addition, optical microscopy was used to determine particle size distribution retained on the surface. The scope of this paper is to explain the initial phase of vacuum cleaner development, including historical Apollo mission data, current state-of-the-art vacuum cleaner technology, and vacuum cleaner testing that has focused on detachment capabilities at varying pressure environments.

\section{Nomenclature}

$$
\begin{array}{ll}
\text { CM } & =\text { Command Module } \\
\text { ECLSS } & =\text { Environmental Control Life Support Systems } \\
\text { ECO } & =\text { Electro-Catalytic Oxidization } \\
\text { ECS } & =\text { Environmental Control System } \\
\text { ESP } & =\text { Electrostatic Precipitators } \\
\text { EVA } & =\text { Extravehicular Activity } \\
\text { HEPA } & =\text { High-Efficiency Particulate Air } \\
\text { ISS } & =\text { International Space Station } \\
\text { IVA } & =\text { Intravehicular Activity }
\end{array}
$$

\footnotetext{
${ }^{1}$ Project Manager, Crew and Thermal Systems Division, EC3, 2101 NASA Rd 1, Mail Stop EC3, Houston, TX 77058, and AIAA Member.

${ }^{2}$ Project Engineer, 2224 Bay Area Boulevard, Houston, TX 77058, Mail Code JE-B21N.

${ }^{3}$ Project Engineer, Crew and Thermal Systems Division, EC3, 2101 NASA Rd 1, Mail Stop EC3, Houston, TX 77058 .
} 


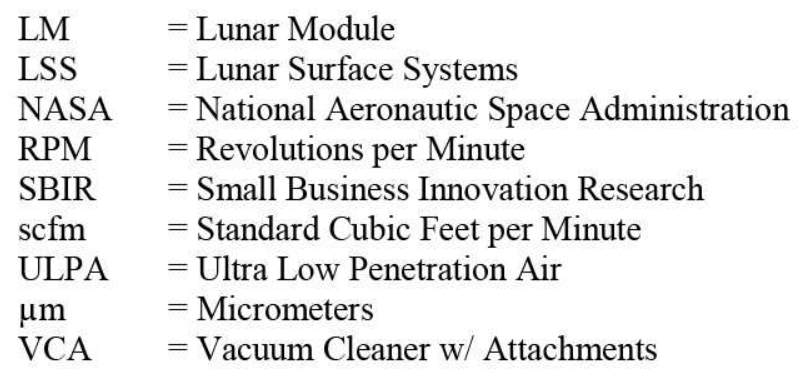

\section{Introduction}

NASA faces a critical need for mitigating the lunar regolith brought into the Airlock and habitable volume by crew members post-extravehicular activities on the lunar surface. Lunar regolith brought in from the lunar surface has a variety of crew health impacts including lung and eye irritation and corrosion, in addition to skin irritation and penetration. However, crew health is most impacted when exposed to respirable lunar dust. Respirable lunar dust accounts for approximately $10 \%$ of lunar regolith particle size ranges which are less than $10 \mu \mathrm{m}$. This respirable lunar regolith can cause respiratory injury in the upper and lower airways causing inflammation, fibrosis and possibly cancer ${ }^{1}$. Lastly, there are still unknown potentially harmful toxicity effects of lunar dust when inhaled due to the chemical composition of the lunar regolith. Crew health impacts due to lunar regolith being brought into the Airlock and habitable volume are not the only concerns. The lunar regolith can cause significant damage to electronics, instrumentation, seals, and environmental control and life support systems (ECLSS) if not appropriately controlled.

Lunar regolith is composed of a variety of chemical properties. Roughly $90 \%$ of the mineral compositions of lunar rocks are Silicate/Aluminosilicates and the remaining $20 \%$ is composed of oxide minerals. Other minor constituents on the Moon include metals such as Iron (Fe), Iron Sulfide (FeS), Nickel (Ni), and Cobalt (Co) ${ }^{2}$. Lunar regolith composition is in the formation of complex, sharp shapes particularly at smaller particle sizes mainly due to space weathering on the Moon's surface. These jagged particles make cleaning fabrics a tedious task, as the particles tend to hook and lock themselves onto the fabric causing an inability to remove particles from the surface. This was experienced during the Apollo program. Apollo crew members complained that lunar dust was not only clinging to the suits, but also penetrating their suits to the point where the dust became a skin irritant $^{3}$.

In order to mitigate lunar regolith in the Airlock and Lunar Surface System's (LSS) habitable volume (cabin), two critical needs have been identified by NASA. The first is the need for an advanced filtration system as a part of the Environmental Control and Life Support System (ECLSS) in habitable volume of LSS, and the second need is for a portable vacuum cleaner for suit and surface cleaning in the Airlock, as well as in the habitable volume of LSS. These two critical needs are to be addressed in series, not in parallel. A crew member would clean themselves to some degree before entering the Airlock (with the use of brushes). Once in the Airlock, a crew member would continue to clean their suit and Airlock surfaces alike with the portable vacuum cleaner. The vacuum cleaner would not only assist the crew member in cleaning the remaining lunar dust off their suit and surrounding surfaces, but also act as an 'air-exchange' for the Airlock, as NASA currently no plans for any filtration system within the Airlock. Once air exchange and suit cleaning are complete, the crew member would be able to enter the habitable volume of LSS with minimal lunar dust contamination. However, any amount of lunar dust that remains would then be captured by the filtration system that will be a part of ECLSS. The main goal of the portable vacuum cleaner is to minimize the amount of lunar dust the LSS cabin is exposed to. This capability becomes increasingly more important as mission duration becomes longer and therefore more time for dust accumulation in the cabin, which could result in damage to critical mission electronics and other intravehicluar (IVA) hardware. 


\section{Background}

\section{A. NASA Vacuum Cleaner (1962 to current)}

A portable vacuum cleaner has been used in many applications in throughout NASA's space program. The first vacuum system known in space was a part of the suit loop on Apollo 11 and 12. The "vacuum brush assembly" was composed of a cleaning hose and vacuum brush. The brush was made from a molded Fluorel Elastomer housing with coarse, flexible Teflon bristles (Fig. 1). The bristles on the vacuum brush were intended for scrubbing the EVA beta cloth suit material to detach and capture the lunar dust that was collected from the lunar surface. The vacuum brush interfaced directly with the vacuum hose, which was attached to the Lunar Module (LM) Environmental Control System (ECS) suit return hose. A 20-mesh filter screen was placed in the molded housing of the vacuum brush to protect the LM ECS line from the entry of large particles.

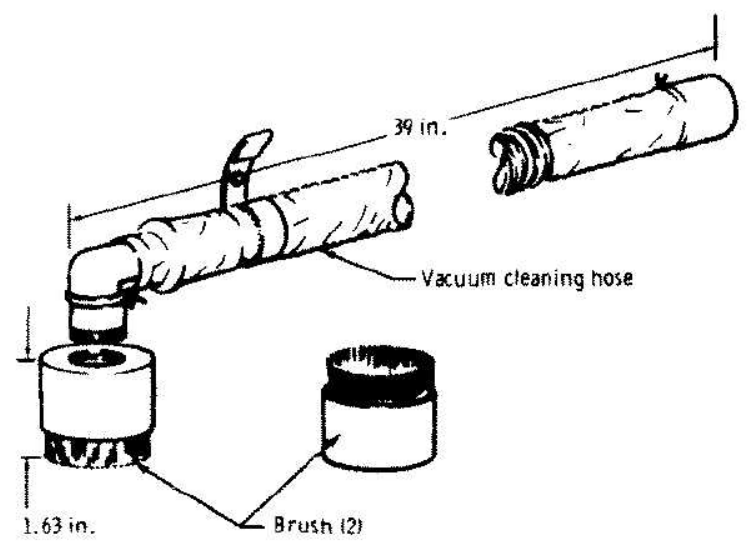

The Apollo 11 crew members reported that they attempted to vacuum their suits, samples and equipment with the vacuum brush assembly before transferring them to the Command Module (CM). With this activity, they reported the clean-up task as tedious, because the suction was too low coming from the LM ECS to detach the remaining lunar dust from the varying surfaces. Apollo 12 crew member, Conrad, reported similarly by expressing the tediousness of the task, saying, "We tried to vacuum clean each other down, which was a complete farce. In the first place, the vacuum didn't knock anything off that was already on the suits. It didn't suck anything, but we went through the exercise." ${ }^{\prime 4}$

The Vacuum Brush Assembly used in Apollo 11 and 12 missions was found to be inadequate in detaching and capturing the lunar dust brought in from the lunar surface. The dust was too fine and suction was too low for the vacuum cleaner to be effective in removing particulate from their EVA suits. In addition, the insufficient design allowed lunar dust to be collected and recirculated in the LM ECS air/ventilation system, which could have caused crew health effects. Although, it remains unconfirmed if crew members experienced health problems related to this.

Once NASA engineers realized that the Apollo 11 and 12 Vacuum Brush Assembly was inadequate, the design was altered for future space flight missions. For Apollo missions 14, 15, 16 and 17 improved vacuum cleaning equipment and procedures were implemented to help mitigate the lunar dust brought into the cabin. The vacuum cleaner, shown in Fig. 2, was made up of a blower (suit circuit compressor), a brush attached at the compressor inlet, hose, a detachable bag made from Armalon felt, and power cord. The vacuum cleaner removed loose dust from the suits and equipment and was also used for cleanup in the command module. Astronaut Schmitt commented that, "The suits were noticeably cleaned by the vacuum cleaner. You could tell you were pulling stuff off them, although they were still dirty. I think most of the dust was taken off." 4

Apollo 16 crew members documented that the vacuum cleaner had clogging problems. The inlet

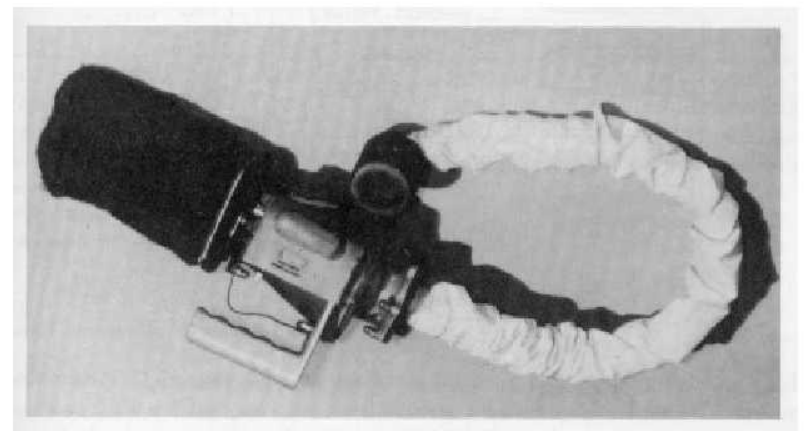

Figure 2. Apollo Portable Vacuum Cleaner screen and impeller required frequent cleaning to remove dust build-up. Specifically, on the Apollo 16 mission, "The vacuum cleaner failed after becoming clogged with dust. The vacuum cleaner was cleaned post-flight and it operated properly. The design of the vacuum cleaner is such that lunar dust can clog the impeller"4 
Currently on the International Space Station (ISS), a wet/dry vacuum cleaner was developed and is used for housekeeping purposes. The vacuum is powered by 120 volts DC. The Vacuum Cleaner Assembly (VCA) can collect both wet (up to $12 \mathrm{oz}$ ) and dry debris (up to 100 cubic inches) utilizing disposable bags, see Fig. 3. The bags act as the first level of filtration and the second level of filtration is a HEPA filter, see Fig. $4 .^{5}$ The HEPA Filter is a square corrugated paper filter in a molded rubber receptacle. The HEPA filter was added to the ISS wet/dry vacuum cleaner to allow filtration of smaller particles. The bag can collect dry debris greater than $6 \mu \mathrm{m}$. The HEPA filter is rated to capture small particles down to $0.3 \mu \mathrm{m}$. A Nomex tool pouch contains vacuum cleaner attachments, which include a crevice tool, brush tool, flexible modular tool and surface tool. The vacuum accessories that are not stowed in the bag include a hose cleaning tool and a suction hose. ${ }^{5}$

The primary use of the VCA is to remove wet and dry non-toxic debris from air filters, mesh screens and other surfaces on ISS. The ISS vacuum cleaner weighs less than 12 $\mathrm{lbs}$, accommodates $100 \mathrm{in}^{3}$ of wet/dry debris or $3 \mathrm{lbs}$ of wet/dry debris. The VCA motor housing is separate from the bag area to ensure that the motor and electronics are not exposed during bag change out. ${ }^{5}$

The VCA's blower is driven by a DC brushless motor, which was intended to reduce the possibility of sparking in the high oxygen environment of ISS. The vacuum has an on/off switch that controls power and the high/low setting for the motor which can run at approximately 11400 RPM and 10700 RPM respectively. During nominal operating conditions on Earth $\left(14.7 \pm\right.$ psia, $\left.72.5^{\circ} \mathrm{F} \pm 7.5^{\circ} \mathrm{F}\right)$ the VCA has the capability of maintaining a minimum sealed suction pressure of 21 inches of water (static pressure).Lastly, the decibel noise level of the VCA is below $80 \mathrm{db}(\mathrm{A})$ measured $3 \mathrm{ft}$ from the source. ${ }^{5}$

Since the VCA has been on the ISS, a few problems have been encountered with the hardware. The first problem

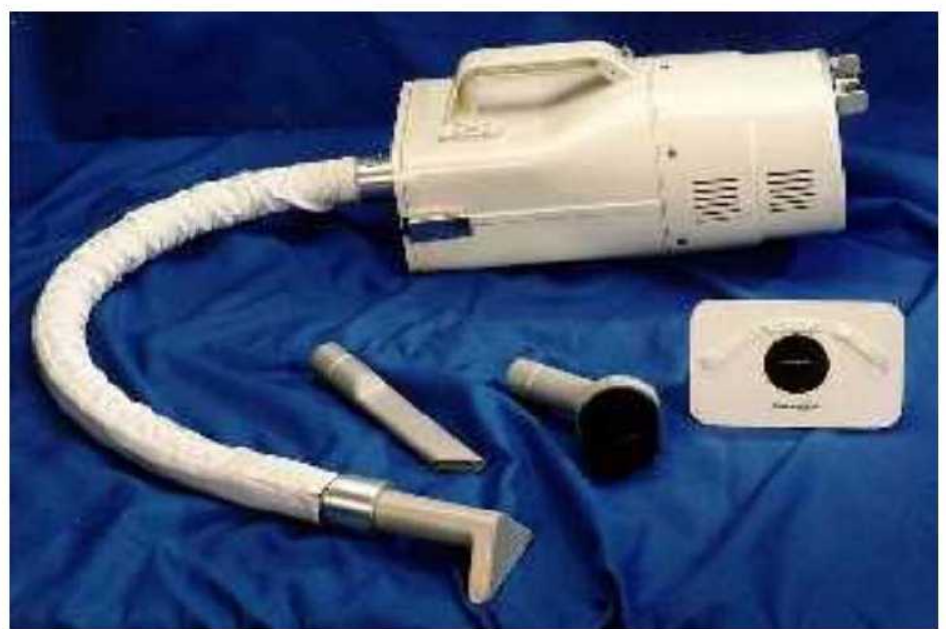

Figure 3. ISS Vacuum Cleaner w/ Attachments (VCA) and Bag

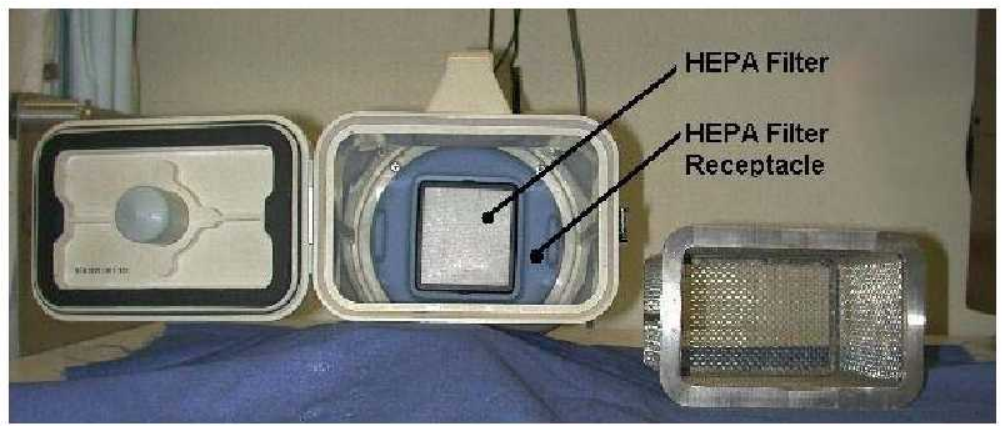

Figure 4. HEPA Filter inside VCA was with the power cable having exposed wires at the connector. This was due to applied strain to the power cable by crew members over time. During the on-orbit repair of the vacuum cleaner cable two design flaws were discovered in the vacuum cleaner: one in the circuit design and one in the electrical bonding interface design. It was found that the vacuum does not have two fault tolerances for the catastrophic hazard of electrical shock. Fault tolerance is required by the Safety and Mission Assurance Community at NASA for this type of electrical flight hardware. The Shuttle and ISS vacuum cleaners were not designed to be compatible with lunar dust. As such, the future vacuum cleaner design for LSS will need to have the ability to mitigate dust size ranges down to the nanometer size and heavy particulate loading.

The Space Shuttle vacuum cleaner is physically the same and performs the same as the ISS vacuum cleaner. The only difference between the two assemblies is power: the space shuttle vacuum cleaner is powered by 28 volts, while the ISS vacuum cleaner is powered by 120 volts. 


\section{B. State-of-the-Art Vacuum Cleaner Technology}

Current state-of-the-art Vacuum Cleaner technology consists of a variation of what the baseline of vacuum cleaner technology has been for years. The vacuum cleaner consists of a few key variables that have been continuously improved on over the years, ever since the first motor powered vacuum cleaner was developed by John Thurman in 1899, followed by the first electric powered vacuum cleaner by Nilfisk in $1910 .^{6}$ Among these variables, power draw, blockage of air passage, filtration, and size of opening of the intake port comprise of the most prevalent areas of vacuum cleaner technology development over the last century.

The 1900's vacuum cleaner was both an upright and canister design which utilized a porous bag for dirt collection. However, in the 1980's Dyson created a vacuum without the canister and standard filtration that was predominant in 1900's. The cyclone design also is referred to as inertial separation technology. The Dyson vacuum cleaner worked by using centrifugal force to separate the dust from the air and by pushing all of the dust into one or more cylinders in a high velocity spiral path, which collected at the bottom of the cylinder. The improvement the Dyson vacuum made was that bag replacement was not necessary, and the suction did not decrease as more dust was collected. These inertial separators are known to this day for their rugged designs, reliability and maintainability.

In parallel with the discussion of vacuum cleaner development, filtration is a critical part of this technology. Currently, there are several different types of filters that exist on the market today. Filters are commonly used in dust-capturing applications; as a result, there are many filter possibilities. Filters vary in construction; some are panels, while others are bags or pockets, and they may be pleated or unpleated. Filters also vary in capacity and efficiency, evaluation parameters used in filter selection. High Efficiency Particulate Air (HEPA) filters are commonly used in commercial vacuum cleaners and filtration systems as a last stage because they are able to remove the smallest particles. HEPA filters are rated as 99.97 percent efficient in the removal of $0.3 \mu \mathrm{m}$ size particles. Ultra Low Particulate Air (ULPA) filters, which exceed HEPA filtering capabilities, remove 99.999 percent of $0.01 \mu \mathrm{m}$ to $0.02 \mu \mathrm{m}$ particles. HEPA and ULPA filters are the most commonly found filters in vacuum cleaners today. HEPA and ULPA are categorized under non-charged filters, while 2 other categories of filters exist, charged and cleanable filters. Nano-filters are new technology of filtration that has been on the rise in the last few years. These filters are in the early stages of development and may be capable of filtering even smaller particles than those filtered by HEPA and UPLA filters. There are a few Small Business Innovation Research (SBIR) contracts for work on the further development of nanofilters. ${ }^{7}$

Along with the standard vacuum cleaner technology development, other technologies have also been developed to collect dust, such as electrostatic precipitators, electrospray gettering, and electro-catalytic oxidiziation. Electrostatic precipitators (otherwise known as ESPs) remove dust from the air by electrically charging the dust particles and collecting them on electrically charged plates. This technology has been implemented effectively in power plants to remove particulates from the air. When properly maintained, ESPs are effective in removing particles down to 0.01 micrometer. Cleaning of the collection plates is essential; if not properly cleaned and maintained, the charged particulates will bypass the plates (and the filtration). However, there are a few of critical concerns for this technology. Specifically, ESPs require high voltage which would be in an oxygen-rich environment on LSS. A failure of a dielectric or insulator would result in sparking, which could lead to combustion in a high $\mathrm{O}_{2}$ environment. ${ }^{8}$ An additional concern for this technology is its ability to produce ozone and the resulting harmful effects of ozone.

Electrospray gettering technology is not yet commercially available. The project is in phase II of development, and is being developed under a NASA SBIR contract with Connecticut Analytical Corporation located in Bethany, CT. According to the Phase II proposal, electrospray gettering is a variant of electrostatic precipitation that does not produce ozone. The electrospray gettering system is intended to be lightweight, collect efficiently, and uses little power.

Lastly, Electro-Catalytic Oxidization (ECO) technology is sometimes used by power plants to remove particulates from the air for pollution control. ECO is similar to ESP, but uses water in its filtration process. Currently, this technology has been applied only in the coal-fired power-plant industry; there is no indication that $\mathrm{ECO}$ has been investigated for use in a portable system, such as a vacuum cleaner. The particles removed using this technology are pollutants from coal [nitrogen oxides $\left(\mathrm{NO}_{\mathrm{x}}\right)$, sulfur dioxide $\left(\mathrm{SO}_{2}\right)$, fine particulate matter $(\mathrm{PM} 2.5)$, sulfuric acid, hydrofluoric acid, mercury, and hydrochloric acid]. The chemical composition of these particles is much different than the chemical composition of lunar regolith. 


\section{Technology Development of a Modified Lunar Surface System Vacuum Cleaner}

The vacuum cleaner technology development for lunar surface systems started in 2009. The project's plan is to study the technology development in 3 different areas over the course of the next 3 years: detachment, transfer and capture. Each area consists of its own technology challenges in the unique lunar surface system environment of reduced pressure and 1/6g lunar gravity. As such, in 2009-2010 the project focused on the first area of technology development of the vacuum cleaner, detachment. The reason the project team has decided to focus on detachment was to investigate the unique adhesion forces that will need to be overcome in order to detach the particles: solar activation and mechanical 'grabbing' of the jagged lunar dust.

The team focused on detachment characteristics of lunar dust simulant, which was tested in three different environment pressures: $14.7 \mathrm{psia}, 8.3 \mathrm{psia}$, and $10.2 \mathrm{psia}$. The reduced pressures of 8.3 psia and $10.2 \mathrm{psia}$ reflected

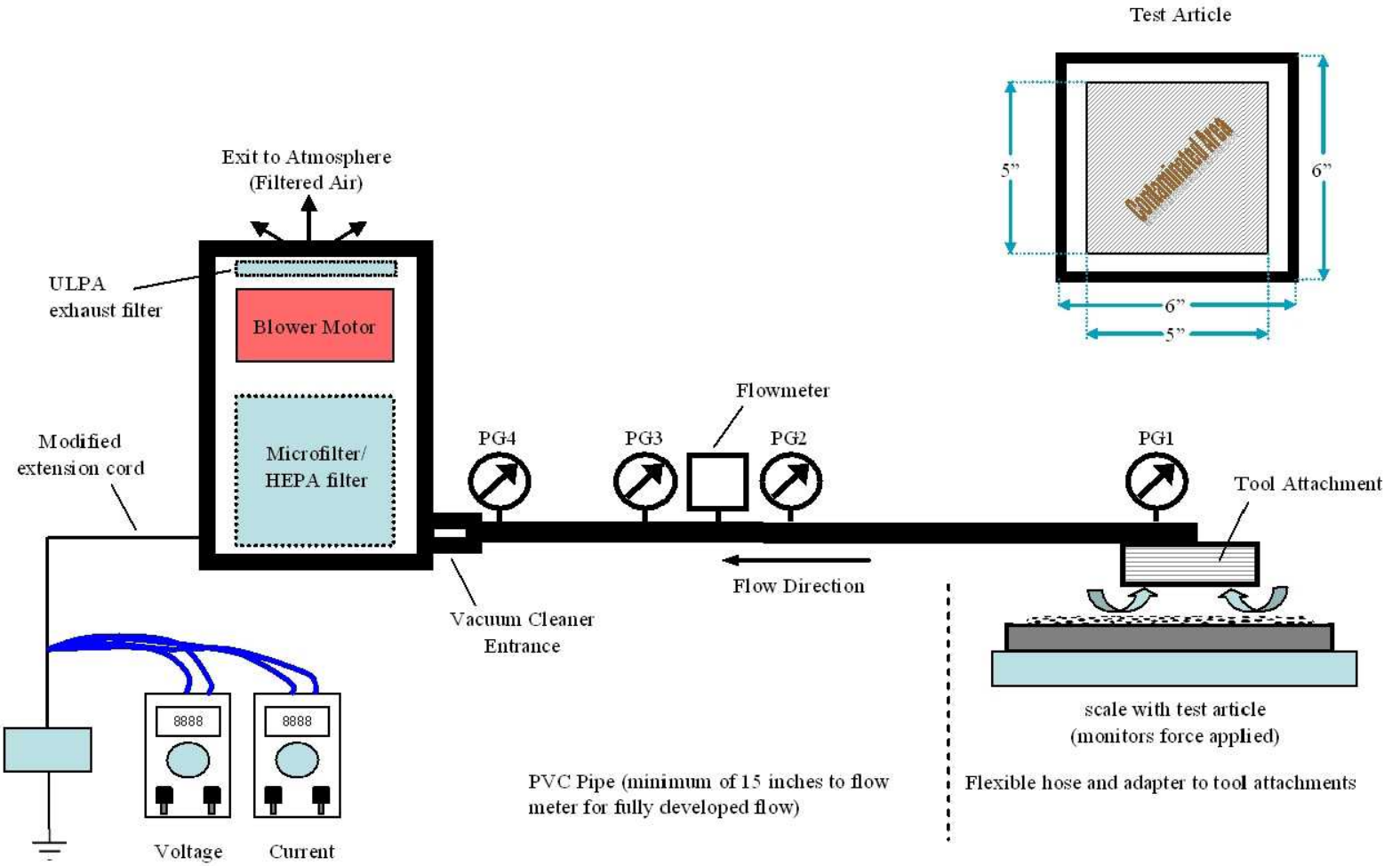

Figure 5. Test Setup Schematic

the pressures that would be seen in the habitable volumes while on the lunar surface. For all testing conducted, the project used a lunar dust simulant that was available to the project during the time of development, JSC-1 AF, made by Orbitec. JSC-1 AF is made from volcanic ash and crushed basalt found in Flagstaff, AZ.

\section{1 ATM Functional Testing}

\section{A. Test Objectives and Setup}

The main objective of this test was to evaluate the performance of a vacuum cleaner using lunar dust simulant, evaluate detachment characteristics of the vacuum cleaner, and to obtain baseline data on which to base development of future testing.

The test setup schematic is shown in Fig. 5. A test article, with lunar dust simulant, was located on a scale, which was used to monitor the force applied to the test article. A vacuum cleaner (see Fig. 6) was used to provide the vacuum source to remove particles from the test article. Various interchangeable tool attachments were attached at the end of the vacuum hose, where simulant entered the vacuum cleaner system. Simulant was carried by the air to vacuum

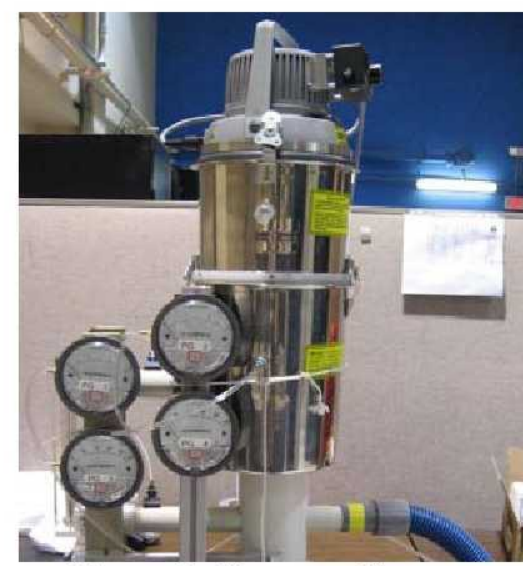

Figure 6. Vacuum Cleaner 
cleaner and captured by a microfilter and HEPA filter. The effects of various parameters on cleaning performance were evaluated. These parameters included cleaning tool, cleaning technique, material to be cleaned, and flow rate. The various tools evaluated during the test are listed below.

- Vacuum Attachments

- Dust Brush

- Utility Hand Tool (no bristles)

- Utility Hand Tool (bristles)

- Bulk Pick-up Nozzle

- Other Tools

- Microfiber Capture Cloth

- Huggies ${ }^{\circledR}$ Wet Wipes

Three cleaning techniques were used with the tools: 1) direct suction grid pattern, 2) unidirectional cleaning pattern, and 3)

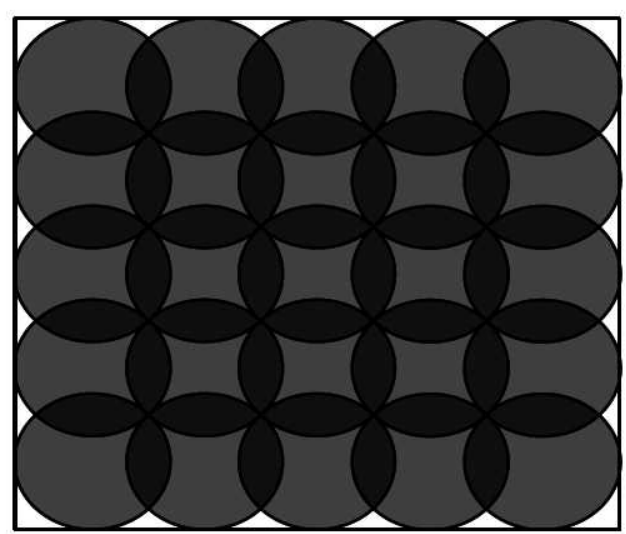

Figure 7. Direct Suction Grid Pattern bidirectional cleaning pattern. Each of these techniques are described in detail in the next section.

Materials, from which dust was cleaned, were chosen to represent a sampling of materials that could possibly be used on a lunar mission. Table 1 provides information for these test materials.

JSC-1 AF was the lunar dust simulant chosen for use in this test. Based on discussions with engineers at Marshall Space Flight Center, the recommended choice for dust simulant was either JSC-1AF or NU-LHT-1D. These two simulants were determined to be best for testing due to their composition (they contain crushed glass materials) and smaller size distribution. These glass-bearing simulants more adequately represented the size distribution and abrasiveness of lunar regolith than the alternative "earthlike/mineral" dusts. JSC-1AF was ultimately chosen as the simulant to use for this testing due to its better cost and availability.

\section{B. Cleaning Techniques}

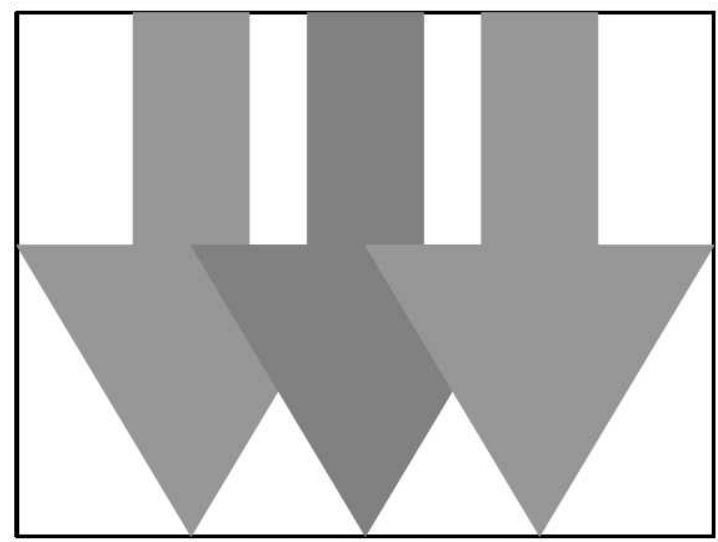

Figure 8. Unidirectional Cleaning Pattern

The three cleaning patterns used during this test were direct suction grid pattern, unidirectional cleaning pattern, and bidirectional cleaning pattern. For the direct suction method, the cleaning tool attachment was placed perpendicularly onto the surface of the test article, then lifted and moved to the next location as illustrated in Fig. 7. This was repeated until the entire surface of the test article had been cleaned.

For the unidirectional cleaning method, also called "scraping", the cleaning tool attachment was placed perpendicularly onto the surface of the test article. Starting at one edge of the test article, the tool was pulled to the opposite edge of the test article. If the tool attachment did not cover the entire surface of the test article, it was lifted and moved to an un-cleaned area, where the motion was repeated until the entire surface of the test article was cleaned by the tool (as illustrated in Fig. 8).

For the bidirectional cleaning method, also called "scrubbing", the cleaning tool attachment was placed perpendicularly onto the surface of the test article. Starting at one edge the test article, the tool was manually pulled to the opposite edge and then pushed back to the first edge for a total of 2 passes. If the tool attachment did not cover the surface area, the tool was lifted and moved to an un-cleaned area where the motion was repeated until the entire test article surface was cleaned (as illustrated in Fig. 9).

Table 1. Properties of Test Materials

\begin{tabular}{|l|c|l|}
\hline \multicolumn{1}{|c|}{ Material } & Part Number & \multicolumn{1}{c|}{ Composition } \\
\hline Orthofabric & 116 & $\begin{array}{l}\text { 400 Denier Gore-Tex/ 200 Denier } \\
\text { Nomex/ 400 Denier Kevlar }\end{array}$ \\
\hline GORE-TEX & VG0180 & Expandable PTFE Teflon yarns \\
\hline GORE-TEX & V112671 & Expandable PTFE Teflon yarns \\
\hline Beta Cloth & CF 250F & Beta Fiberglass with PTFE coating \\
\hline Velcro & 190995 & Nomex yarn \\
\hline Aluminum & 2025 & n/a \\
\hline Composite Laminate & $\begin{array}{c}\text { AS4/3501-6, } \\
108 G L / 3501-6\end{array}$ & $\begin{array}{l}\text { 8 layers carbon laminate, 2 layers } \\
\text { (outer) fiberglass }\end{array}$ \\
\hline
\end{tabular}




\section{Test Procedure}

Each test article was prepared by being pulled taut to a wooden frame. All test articles were then weighed and photographed at three times during testing: 1) before being soiled (new), 2) after being soiled, and 3) after being cleaned with the vacuum cleaner. After the first weighing and photograph, the test article was then secured to the scale for cleaning. A measuring spoon (1 teaspoon) was used to measure and apply JSC-1AF to the test article. Figure 10 shows an example of a dirtied test article. A thick layer of simulant was spread across the test article. This overloading of the test article was done in order to be conservative and to fully saturate the surface.

The test articles were cleaned with varying test parameters (cleaning tool, cleaning technique, material, and flow rate). Then cleaning performance was evaluated by measuring the amount of simulant remaining on the material.

\section{Data Analysis}

Photographs taken after cleaning of a test article illustrated that dust particles had a tendency to "jump" after the utility tool (without bristles) was removed from the fabric during the direct suction method cleaning test. This "jumping" effect left visible lines of dust on the Orthofabric (see Fig. 11).

Photographs were also used to illustrate that wet wipes and microfiber cloths were not very efficient at removing dust from Orthofabric. It was observed that the wipes seemed to smear the dust on the fabric instead of removing it (see Fig. 12 and Fig. 13). This observation was supported by mass data, which indicated that the test points using vacuum cleaner attachments left the least amount of simulant remaining on the test materials. This emphasized the need for an alternative cleaning technique, such as vacuum cleaning.

Analysis of the mass results indicated that the tool with the smallest opening, the nozzle tool, performed more effectively than other tools. The data in Fig. 14 shows that the nozzle tool left the least amount of simulant on the materials tested. Differences in the performance of the other tools could not be determined.

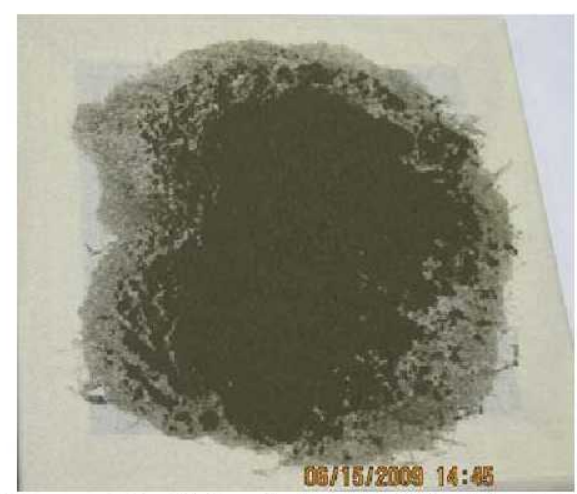

Figure 10. Fully Saturated Test Article

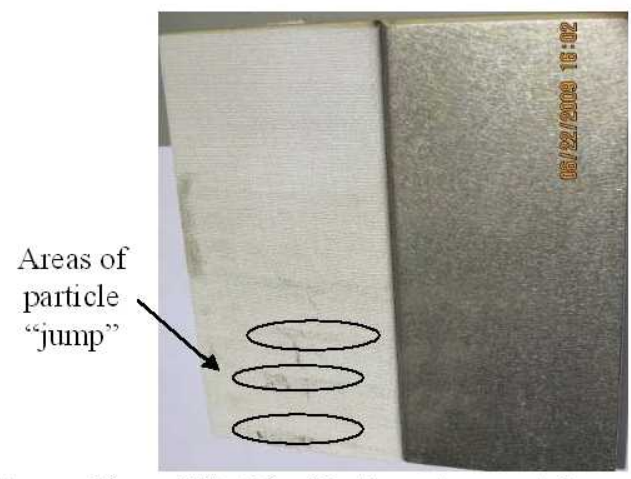

Figure 11. TA-66 (Ortho to metal seam, utility tool (no bristles), direct suction, $15 \mathrm{cfm}$ )

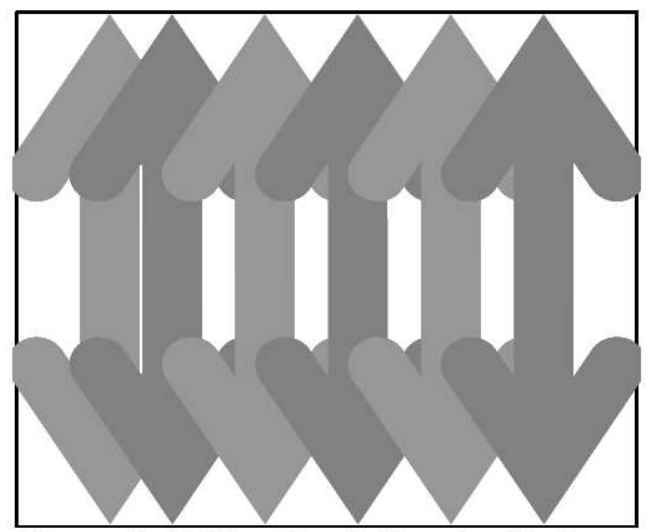

Figure 9. Bidirectional Cleaning Pattern 


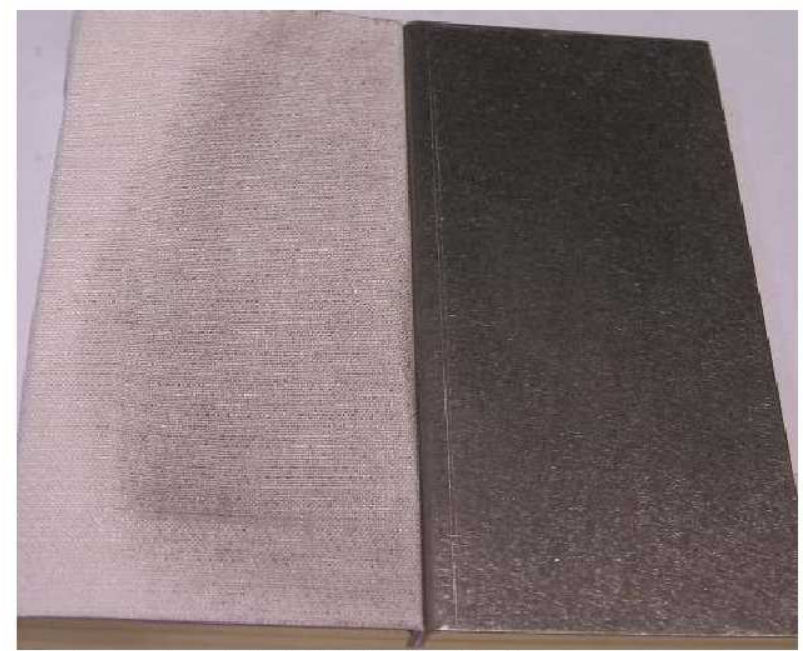

Figure 12. TA-103 (Orthofabric to metal seam, microfiber cloth, scrubbing)

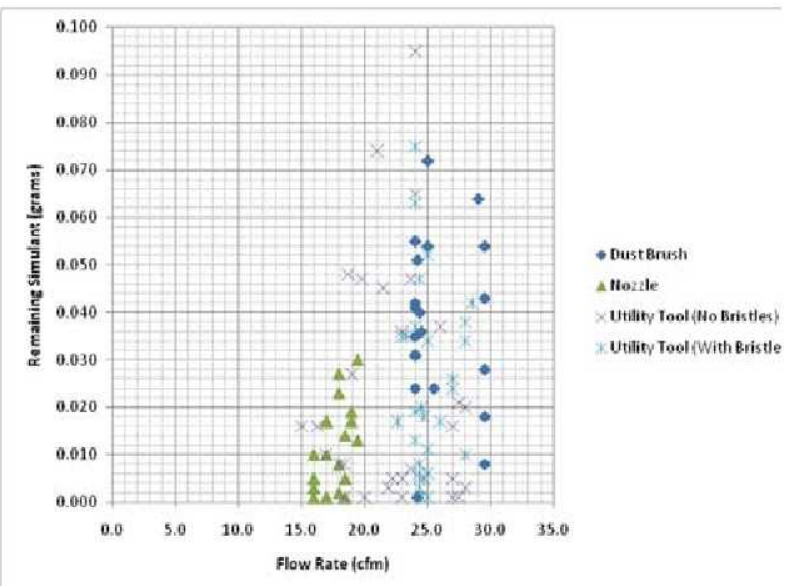

Figure 14. Mass Data Organized by Tool Type, Velcro Data Points Excluded, Cloths Excluded

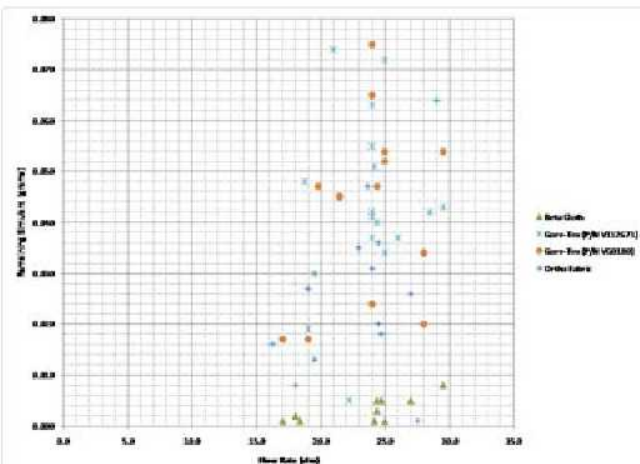

Figure 16. Mass Data Organized by Material, Filtered for Beta Cloth, Gore-Tex, and Ortho Fabric, Excludes Microfiber and Wet Wipe Tools

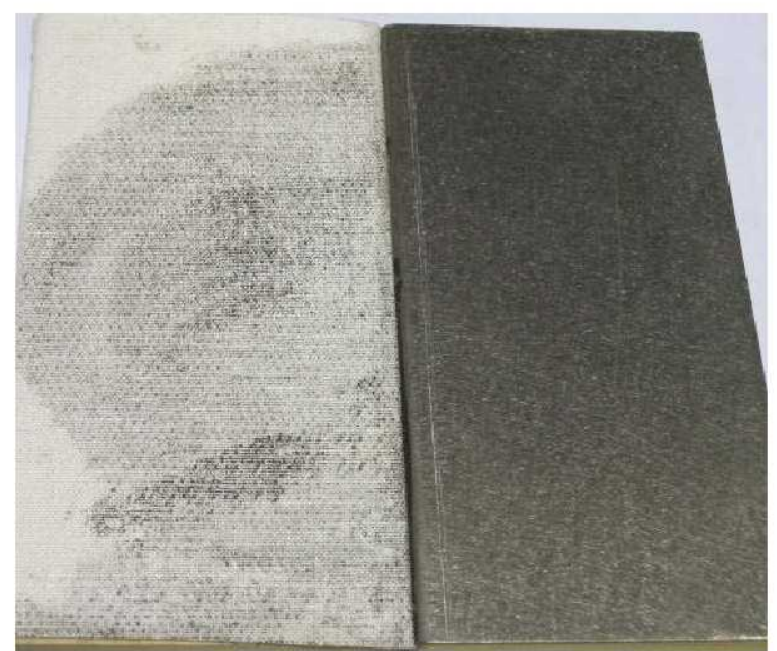

Figure 13. TA-52 (Orthofabric to metal seam, wet wipes, scrubbing)

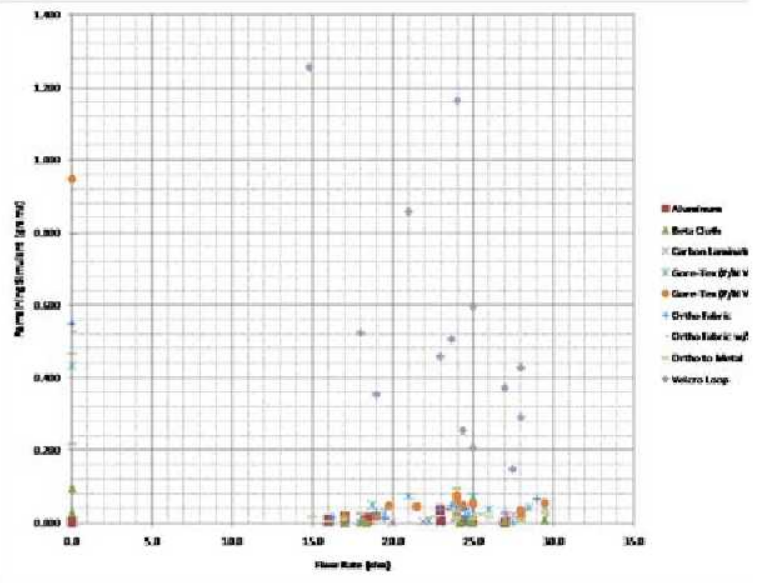

Figure 15. Mass Data Organized by Material

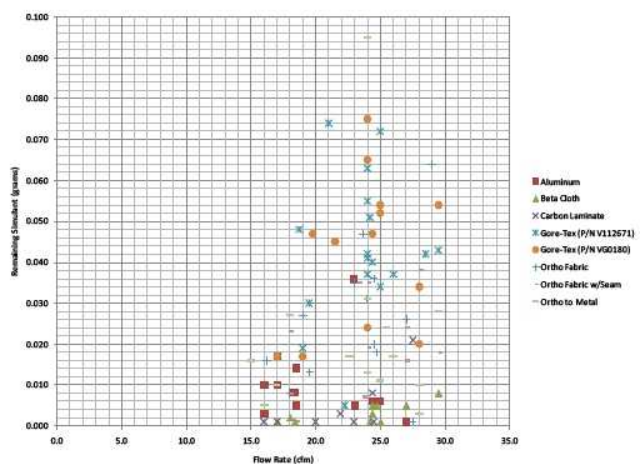

Figure 17. Mass Data Organized by Material, Excludes Velcro, Excludes Microfiber and Wet Wipe Tools 
showed that the mass remaining for test points with Velcro was an order of magnitude higher than the simulant remaining for tests with other materials (see Fig. 15). Accumulation of dust in Velcro would need to be avoided or a special method of removal developed.

The mass data shown in Fig. 16 shows that the beta cloth was better than the other materials. However, the mass remaining on the Gore-Tex and Orthofabric were similar.

Review of the data in Fig. 17 showed that it was easier to remove simulant from aluminum and carbon laminate when compared to most materials, as expected. However, the performance was not much better than that of beta cloth.

It was expected that, in general, less simulant would remain on materials that were cleaned with higher flow rates. These hypotheses were supported by the fact that higher flow rates would mean higher drag forces to remove the particles. However, the results varied too greatly with respect to flow rate, and no general trends could be identified. Therefore, conclusions could not be made on any hypotheses regarding flow rates. The cause for this was assumed to be uncertainty in the mass scale. Therefore, photomicrograph image analysis was used as a more accurate method of obtaining results. Unfortunately only a small sample set of test points were analyzed and general trends could not be inferred.

\section{E. Lessons Learned from 1 ATM Testing}

Human error was identified as a major source of variability between tests. The pressure applied to the test article was neither recorded nor controlled, resulting in varied applied pressure between test operators. The human test subject introduced further variability by not consistently controlling the angle between the tool attachment and test article. To control this variability, a two axis nozzle control apparatus was chosen to be utilized in follow-on testing. This apparatus had four main components: dolly, bogen arm, track, and clamp. The dolly allowed for the movement of the apparatus along the track. The bogen arm attached to the dolly on one end and the clamp on the other. By tightening the joints of the bogen arm and the clamp, the operator variability was expected to be drastically reduced.

Mass measurements were initially used to compare the simulant remaining on the cleaned test articles. During post-test analysis, it was determined that significant differences between most test points could not be determined, partially due to the scale uncertainty and the size and mass of particles that were being evaluated. Therefore, only an incomplete analysis of the data could be made. For example, trends with respect to flow rate could not be made. This accentuated the need for a more precise method of quantifying the particles remaining on the test surfaces in order to properly analyze the results.

\section{Reduced Pressure Testing}

\section{A. Test Objective and Setup}

The main objective for the second phase of testing was to determine the effects of a reduced pressure environment on the removal efficiency and detachment characteristics of the simulant. It is projected that the LSS Airlock will be pressurized to 8.3 psia and the habitable volume on the LSS vehicle will be pressurized to 10.2 psia. Knowing if the removal efficiency of the vacuum cleaner changed at reduced pressures would be very valuable knowledge. If the removal efficiency is not affected by reduced pressure, further testing could be performed at standard atmospheric conditions and considered analogous to the results that would be seen in the Airlock and habitable volume. This would avoid complex and costly testing Figure 18. Test Facility that would yield results similar to those obtained in a standard atmospheric environment.

The test setup for the reduced pressure test was similar to that of the 1 ATM functional test. The major changes between the test setups were the inclusion of the additional equipment deemed necessary for the reduced pressure test by the lessons learned from the 1 ATM test. The reduced pressure testing was performed in the 11 Foot Outer Lock Vacuum Chamber (Fig. 18) and was completed early 2010. 


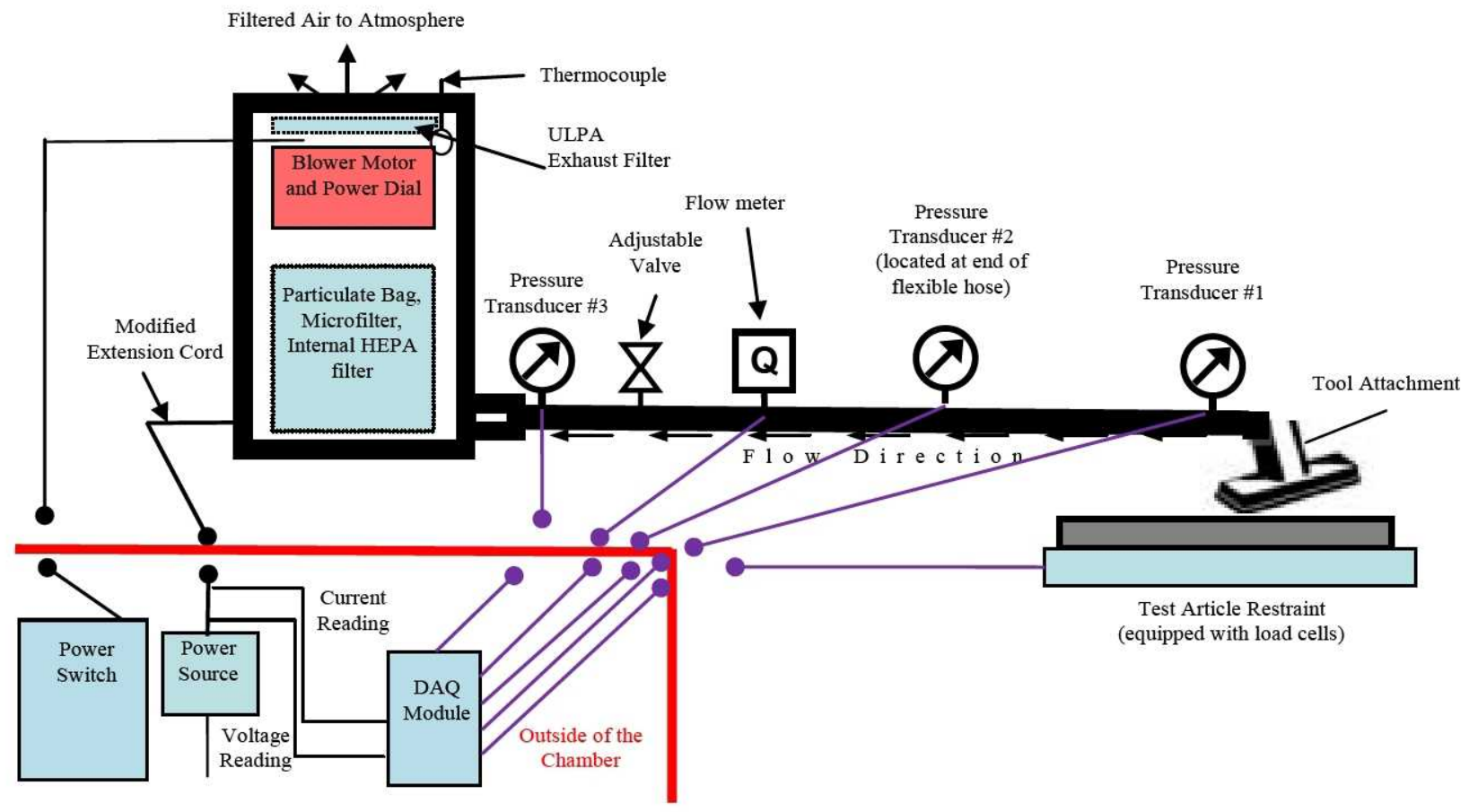

Figure 19. Reduced Pressure Test Setup

It is important to note that there were differences between the test setup for 1 ATM functional testing and reduced pressure testing. Some of these significant changes and their drivers are listed here (Fig. 19). The first major difference was the inclusion of an adjustable hand valve. During the 1 ATM test, only three flow rate settings were used due to the limitations of the vacuum cleaner. By adding the adjustable hand valve in the reduced pressure test setup, the range of achievable flow rates was greatly increased. The second major difference was a change in data acquisition methods. For the 1 ATM test, data was not recorded dynamically. For the reduced pressure test, instrument readings were recorded once per second. The 1 ATM test lessons learned resulted in the inclusion of a bogen arm and dolly during the reduced pressure testing to allow for control over the orientation and pressure of the tool attachment (Fig. 20).

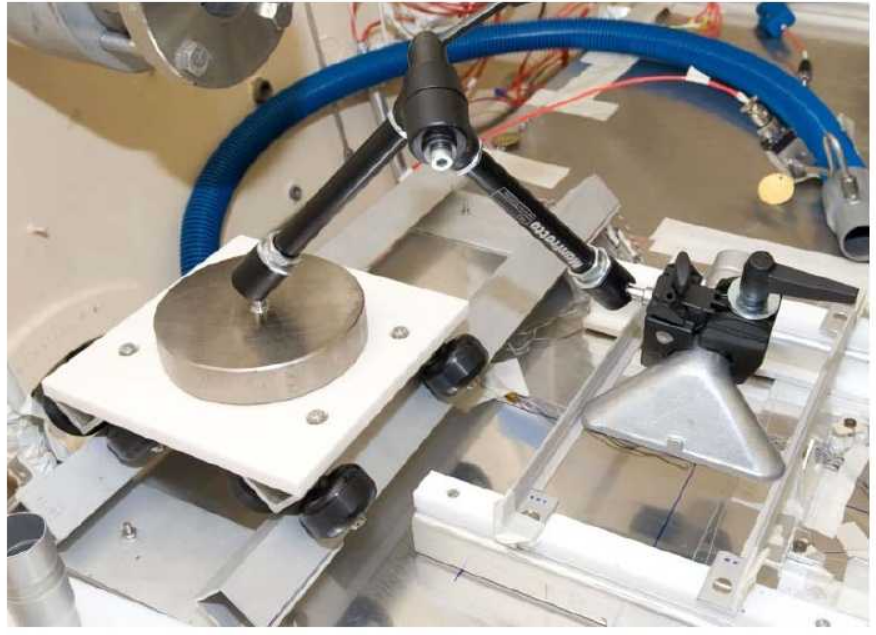

Figure 20. Bogen arm and dolly

\section{B. Test Variables}

For the reduced pressure test, six variables were tested. The first variable was the test article material. Orthofabric and GORE-TEX ${ }^{\circledR}$ are potential space suit materials that will be worn during Extravehicular Activities (EVAs) on the lunar surface. For this purpose, they were the two test article materials chosen for this test. The second variable that was tested was the permeability of the test article materials. Space suits worn on EVAs have a low permeability by design so it was important for the team to determine if the permeability of the test article affects the removal efficiency. To decrease the permeability of appropriate test points, an aluminum backing was applied under the test article to prevent air flow from underneath the test article. The third variable was the flow rate setting of the vacuum 
cleaner. Flow rates of $15 \mathrm{scfm}, 30 \mathrm{scfm}$, and $45 \mathrm{scfm}$ (herein after referred to as low, medium, and high, respectively) were studied in the reduced pressure test. It is important to note that the test points were grouped by flow rate setting, not actual flow rate. Once the tool attachment was applied to test articles with low permeability, the flow rate measurement often decreased below the pre-set value. The fourth variable was the cleaning method used to remove simulant from the test article. The simulant used (JSC-1AF) is a poor analog of the shape of lunar regolith dust. Specifically, lunar dust is jagged, where the JSC-1AF simulant is spherical. Therefore, agitating the simulant with the vacuum attachment on the test article was determined to not be an adequate representation of how actual lunar dust would detach from surfaces on the LSS.

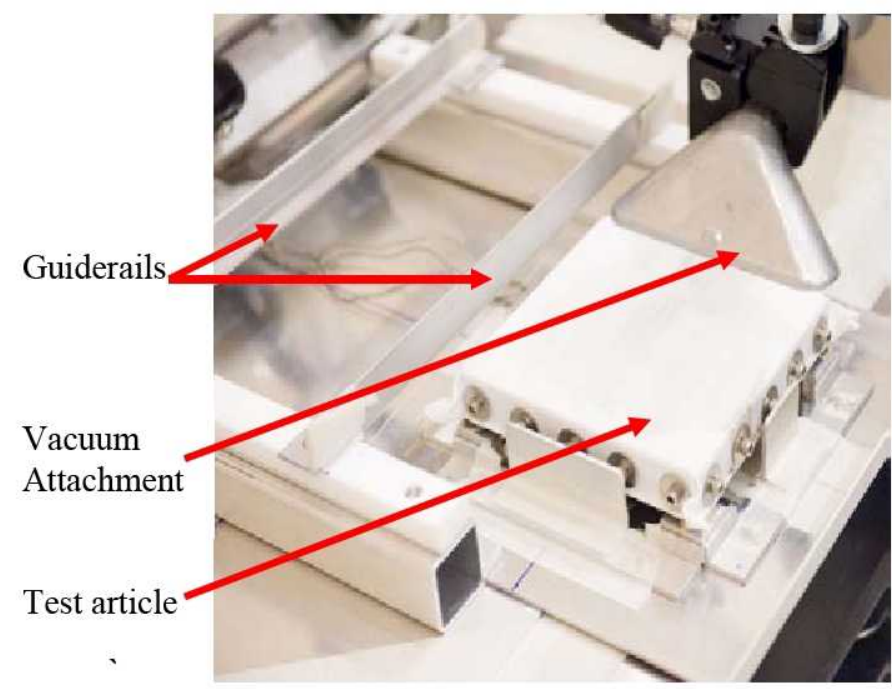

Figure 21. Cleaning Method Illustration As such, this test examined the flow of air over the test articles by incorporating no contact test points. This means that the tool attachment was placed on a guiderail and the test article was cleaned from a regulated height of lcentimeter with no direct contact between the tool attachment and test article. The second cleaning method involved direct contact between the tool attachment and test article. Fig. 19 shows the tool attachment resting on a test article after a contact cleaning method test point. You can see the guiderail to the left that was used for no contact cleaning method test points. Both cleaning methods involved a bi-directional movement of the tool attachment meaning that the tool attachment covered the test article area two times (see Figure 9). The fifth variable for this test was the tool attachment used to clean the test articles. This test examined the removal efficiency of three different tool attachments: utility hand tool with bristles, utility hand tool without bristles, and bulk pickup nozzle, same tools used in the 1 ATM Functional Testing for comparison. The final variable investigated during the reduced pressure test was the pressure inside of the vacuum chamber. Test points were conducted at the expected pressures of both the LSS Airlock and habitable volume.

\section{Data Collection and Preliminary Results}

As in the 1 ATM test, the simulant remaining on the test article is not the only data of value. The instruments included in this test setup were three pressure transducers, a flow meter, a current meter, a volt meter, a thermocouple, and a load cell. The pressure transducers took measurements along the flow path. The first location was at the junction of the tool attachment and the flex hose line. The second was at the junction of the flex hose line and flow path piping. The final pressure transducers were located at the junction of the flow path piping and the vacuum cleaner. The flow meter was located in the flow path piping between the second and third pressure transducers. The current and volt meters gave readings on the power of the vacuum cleaner. The thermocouple measured the internal temperature of the motor. And the load cell was placed under the test article to measure the amount of force applied on the test article during the test points.

Analysis of the test articles is ongoing and will not be available until mid year 2010. Photomicrographs of the cleaned test articles will be taken using both optical and scanning electron microscopy. Image analysis software will then be used to analyze the photomicrographs to quantify and size particles as small as $0.2 \mu \mathrm{m}$. These results will then be compared to the distribution and quantity of particles initially deposited on the test articles, determined by a similar method.Although data analysis is not complete, some general qualitative observations have been made about the results of the testing in the interim. Significantly less simulant was removed from test points that utilized the no contact cleaning method than those with the contact cleaning method. Of the no contact cleaning method test points, the removal of simulant was best for the high flow rate setting and worst for the low flow rate setting. Figures 22 , 23 , and 24 show test points that were identical with the exception of the flow rate setting: high, medium, and low respectively (see Table 2 for complete details on test point variables).

The test articles shown in Figs. 22, 23, and 24 were cleaned at a pressure of 10.2 psia. The matching test points at a pressure of 8.3 psia can be seen in Figure 25, 26 and 23. These figures show a similar trend in removal efficiency according to flow rate; the higher the flow rate the better the removal efficiency. Note that the high and medium flow rate setting test articles show greater overall removal efficiency at a lower pressure while the low flow rate 


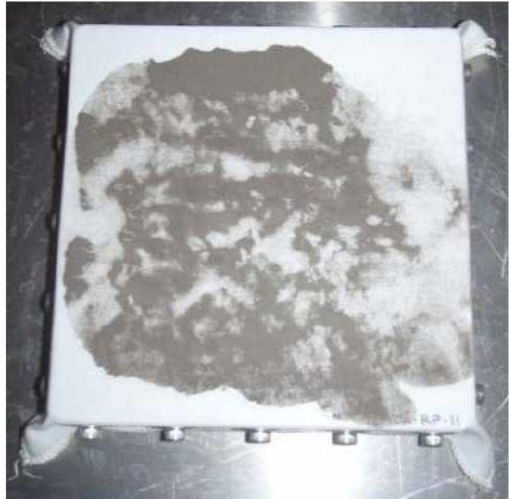

Figure 22: Test Point 11

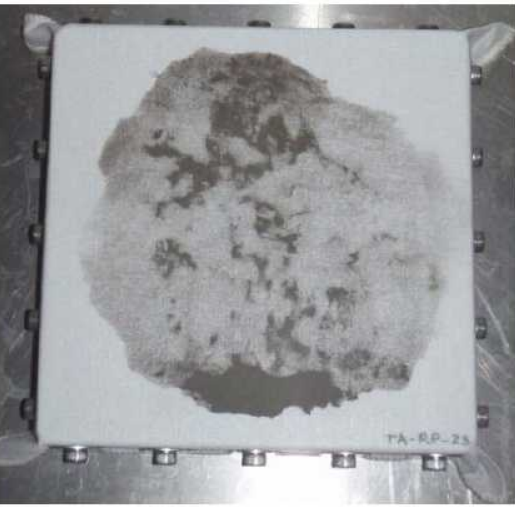

Figure 25: Test Point 23

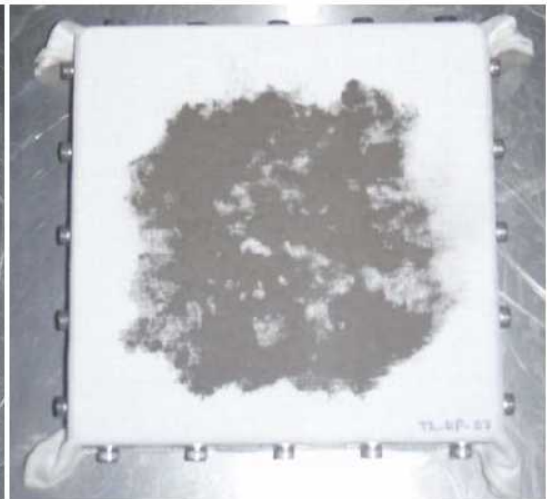

Figure 23: Test Point 7

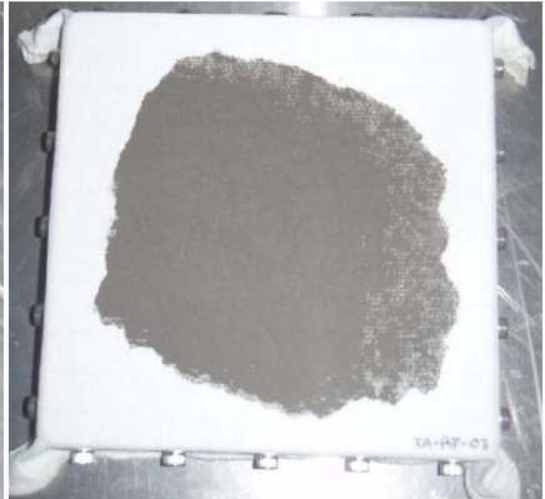

Figure 24: Test Point 3

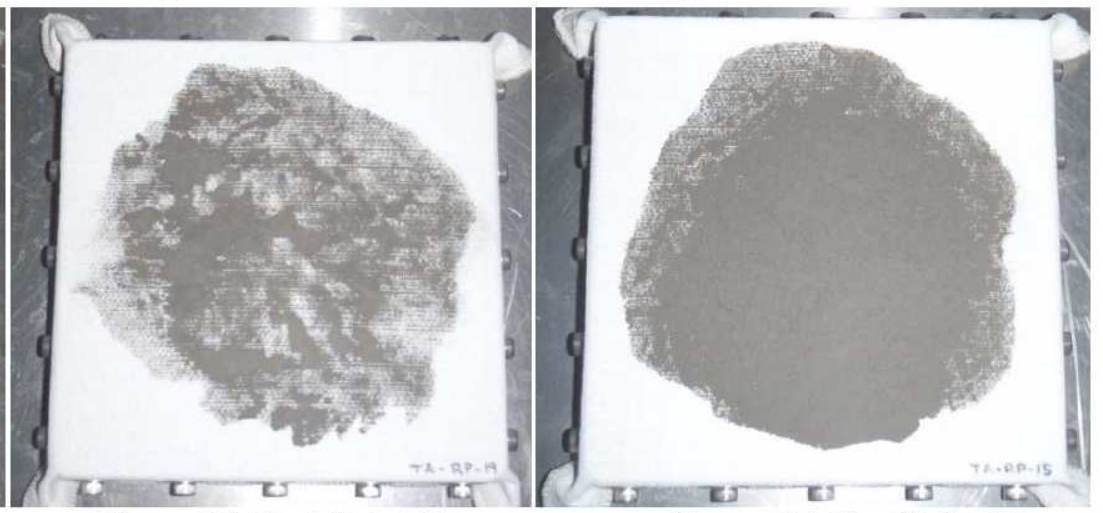

Figure 26: Test Point 19

Figure 27: Test Point 15

setting test article shows no visual change with pressure. The full impact of this will not be known until data analysis is complete.

It was noted that test points with high permeability test articles exhibited more resistance when being cleaned than the test points with low permeability test articles. It is believed that this is likely due to the high permeability of the test article that allows for a more complete 'seal' between the test article and tool attachment, which in turn allows an increase amount of air flow that can move around the particles to help detach them from the surface.

It was noted that simulant collected in the bristles of the hand tool with bristles. This means that simulant removed from the test article was not securely contained within the vacuum cleaner and could disperse back into the airlock or habitable volume.

Table 2. Reduced Pressure Abridged Test Matrix (Reference Figures 22 - 27)

\begin{tabular}{|c|c|c|c|c|c|c|}
\hline $\begin{array}{c}\text { Test } \\
\text { Point }\end{array}$ & $\begin{array}{c}\text { Pressure } \\
\text { (psia) }\end{array}$ & Tool & Material & Flow Rate & $\begin{array}{c}\text { Cleaning } \\
\text { Method }\end{array}$ & Permeability \\
\hline 11 & 10.2 & $\begin{array}{c}\text { utility tool } \\
\text { without bristles }\end{array}$ & Orthofabric & High & No contact & Low \\
\hline 7 & 10.2 & $\begin{array}{c}\text { utility tool } \\
\text { without bristles }\end{array}$ & Orthofabric & Medium & No contact & Low \\
\hline 3 & 10.2 & $\begin{array}{c}\text { utility tool } \\
\text { without bristles }\end{array}$ & Orthofabric & Low & No contact & Low \\
\hline 23 & 8.3 & $\begin{array}{c}\text { utility tool } \\
\text { without bristles }\end{array}$ & Orthofabric & High & No contact & Low \\
\hline 19 & 8.3 & $\begin{array}{c}\text { utility tool } \\
\text { without bristles }\end{array}$ & Orthofabric & Medium & No contact & Low \\
\hline 15 & 8.3 & $\begin{array}{c}\text { Utility tool } \\
\text { without bristles }\end{array}$ & Orthofabric & Low & No Contact & Low \\
\hline
\end{tabular}


Data analysis and a full test report for this test are expected to be complete in May 2010. The Materials and Processes Branch will provide three of each kind of image for data analysis. These images are all taken within the innermost 1.5 " by 1.5 " square of the test article. These three locations were chosen to be 1 ) a region representative of the test article, 2) the cleanest region (upon visual inspection), and 3) the dirtiest region (also upon visual inspection). By taking the mean of these three data points, the team hopes to achieve a representative measure of the actual removal efficiency of the test point. The conclusions that are drawn from the results of this test will be very valuable in deciding if all further testing will need to be conducted in a reduced pressure environment to provide useful data for the vacuum cleaner's planned application.

\section{Future Plans}

The vacuum cleaner technology development will continue to research the areas of detachment, transfer and capture, with emphasis on transfer and capture over the next several years. In FY2010, the project plans to retest some of the Reduced Pressure Test Matrix in 1 ATM. The testing will be redone to capture some of the data missing in the first 1 ATM testing that was captured in Reduced Pressure for data comparison and analysis purposes. In addition, the project will research the filter design aspect of the vacuum cleaner design to incorporate into the next round of testing. The choice of filtration could affect the efficiency of the vacuum, which needs to be understood before the vacuum cleaner develops any further. The filter trade study will kick start the projects efforts into the next area of technology development, transfer.

Over the next several years, prototypes will be developed and tested at reduced pressure and then in reduced gravity, as NASA budget allows. Near FY15, a final design will be selected and tested in the integrated lunar surface system design.

\section{Conclusion}

To support the future of manned lunar surface missions, NASA JSC has focused on the area of particulate detachment in efforts of developing a lunar surface system vacuum cleaner. In June 2009, 1 ATM laboratory testing was performed to evaluate the influence of various factors such as flow rate, surface material, cleaning methods, and different tool attachments on vacuum cleaner efficiency. A review of the test data did not indicate any statistical significance amongst all of the variables. However, valuable lessons learned from this testing were utilized in the reduced pressure testing performed in January 2010. The reduced pressure testing data is currently under analysis with the Imagery Lab at NASA JSC. The initial conclusion that can be drawn from the testing was that variability of human interactions was controlled better than before by utilizing a test set up that limited the mobility and angularity of the vacuum cleaner attachment, and therefore the force applied on the test coupon during testing. By removing this variability, statistically relevant data should be obtained from the test.

\section{Acknowledgments}

We would like to thank Erin Brach, Jacobs Technologies, for assisting the development of a lunar surface system vacuum cleaner, specifically during the 1 ATM Testing phase of the project.

\section{References}

${ }^{1}$ Kahn-Mayberry, Noreen. Lunar Dust Workshop (sponsored by Glenn Research Center). Nov 2008. https://collaboration.grc.nasa.gov/eRoom/NASAclfl/DUSTDemoRoom/0 f2fa3

${ }^{2}$ Gaier, James R. Lunar Dust Workshop (sponsored by Glenn Research Center). Nov 2008. https://collaboration.grc.nasa.gov/eRoom/NASAclf1/DUSTDemoRoom/0 f2fa3

${ }^{3}$ J.R. Gaier, "The Effects of Lunar Dust on EVA Systems During the Apollo Missions. "NASA/TM-2005213610/Revl.

${ }^{4}$ Wagner, Sandra. "The Apollo Experience Lessons Learned for Constellation Lunar Dust Management. " NASA/TP-2006-213726, 2006. September 2006.

${ }^{5}$ Toon, Katherine. "Technology Assessment Memo for Lunar Surface System Vacuum Cleaner." ESCG-4110-09IVACP-MEMO-0006. March 20, 2009 
${ }^{6} \mathrm{http}: / /$ en.wikipedia.org/wiki/Vacuum_cleaner

${ }^{7}$ Seldon Technologies, Inc. Proposal \#07-2 X3.01-8838, and, Agave BioSystems Proposal \#X3.01-8268.

${ }^{8}$ Cognata, T., Conger, B., and Paul, H. L., "Results of the Particulate Contamination Control Trade Study for Space Suit Life Support Development," International Conference on Environmental Systems, 2009-01-2373, Savannah, GA, 2009. 